\title{
Letter
}

\section{A Theory on the Birth, Structure and Ultimate Fate of the Universe}

\author{
Hitoshi Shibata ${ }^{1}$, Norio Ogata ${ }^{2, *}$ \\ ${ }^{1}$ Taiko Pharmaceutical Co., Ltd., Osaka, Japan \\ ${ }^{2}$ Rearch Institute, Taiko Pharmaceutical Co., Ltd., Seikacho, Japan
}

Email address:

hshibata@seirogan.co.jp (H. Shibata),nogata7@yahoo.co.jp (N. Ogata)

${ }^{*}$ Corresponding author

\section{To cite this article:}

Hitoshi Shibata, Norio Ogata. A Theory on the Birth, Structure and Ultimate Fate of the Universe. American Journal of Modern Physics. Vol. 5, No. 5, 2016, pp. 142-145. doi: 10.11648/j.ajmp.20160505.14

Received: August 7, 2016; Accepted: August 26, 2016; Published: September 13, 2016

\begin{abstract}
One major theory stipulates that our Universe was born through a process of fluctuation in a quantum world, the Big Bang and inflation, and is still expanding. However, questions concerning dark energy, dark matter and other major issue remain unanswered. If it is assumed that the quantum world remains outside of the Universe in the same state as before the birth of the Universe, and that the Universe is a space-time bubble created therein with a boundary that is an event horizon similar to a black hole, then it can be proven that the Universe is not expanding but contracting from its boundary, while satisfactorily explaining the observed results of the Universe's expansion and isotropy. The authors propose this as a new theory.
\end{abstract}

Keywords: Universe, Big Bang, Inflation, Dark Matter, Dark Energy, Black Hole, Quandom, Cosmic Microwave Background

\section{Introduction}

Since the observation in the 1920 s by E. Hubble confirmed that the Universe is expanding $[1,2]$, it was further found by the observation of the explosion of supernovae of farther way that the speed of the expansion of the Universe is accelerating [3]. However, the nature of "dark energy" that is believed to be accelerating the expansion of the Universe is still unknown [4-9]. If the speed of the expansion of the Universe is actually accelerating, then a hypothesis that can explain the source of energy and the ultimate end point of the expansion may be required. To the best of our knowledge, no hypothesis or observation that can explain such phenomena is found so far in the literature. The hypothesis presented in this paper could explain these unsolved important questions of physics and the fate of the Universe. The hypothesis will further become an important driving force to open novel experiments in cosmology.

\section{Theory and Concept}

\subsection{The Birth and Structure of the Universe}

If our Universe is the result of the Big Bang, let us assume that before our Universe a quantum world with no time or distance existed, and let us call such a world a "quandom." There is no space or time in a quandom, where matter and energy are condensed into a single point in a quantum state with no position or length. A quantum fluctuation there could result in the Big Bang and the subsequent inflation. This is as if a phase transition occurred in boiling water at a temperature higher than water's boiling point, resulting in a big steam bubble in the water. Similarly, if our Universe is regarded as a space-time bubble created as a result of a phase transition in a quandom, it can be considered that the outside of the Universe is a quandom, and that the edge of the Universe is a boundary between the Universe and the quandom containing it, similar to a boundary between water and a bubble. Hereinafter, let us call this boundary between the Universe and the quandom containing it "outer space-time wall".

The outer space-time wall gets part of its energy from the quandom surrounding it, and an event horizon is formed by an 
enormous gravitational force corresponding to the mass equivalent of the outer space-time wall's energy. Similarly, if we assume that the inside of a black hole with an event horizon is a quandom, it can be said that the event horizon of the black hole is an "inner space-time wall." It can be assumed that at the Big Bang, the Universe started expanding inwards from its outer space-time wall, rather than expanding outwards from its center. This is when the space-time of the Universe was formed, and the physical distance and time from the outer space-time wall subsequently came into existence. After having been released and spread around the Universe, matter with mass has been gathered by gravitational forces, while the Universe is simultaneously being pulled back by the gravitational force exerted by the outer space-time wall (Fig. 1).

\subsection{The Expansion and Isotropy of the Universe}

It appears that distant stars are receding from us with tremendous speed, irrespective of direction, and additionally that the speed at which they are receding is increasing. It is possible that the reason for this is because the Universe is being pulled back to the outer space-time wall. In regions far distant from the outer space-time wall, space-time is being pulled back slowly, and so the redshift of stars in the region does not increase very much, creating the appearance that the Universe is expanding at a constant speed there. On the other hand, in regions near the outer space-time wall, space-time is compressed by the outer space-time wall's tremendous gravitational force and matter is pulled back to the outer space-time wall at accelerating speeds, resulting in the observed major increase in the redshift of stars. Because the outer space-time wall exists all around the Universe, it can be observed from anywhere in the Universe that the Universe is expanding isotropically.

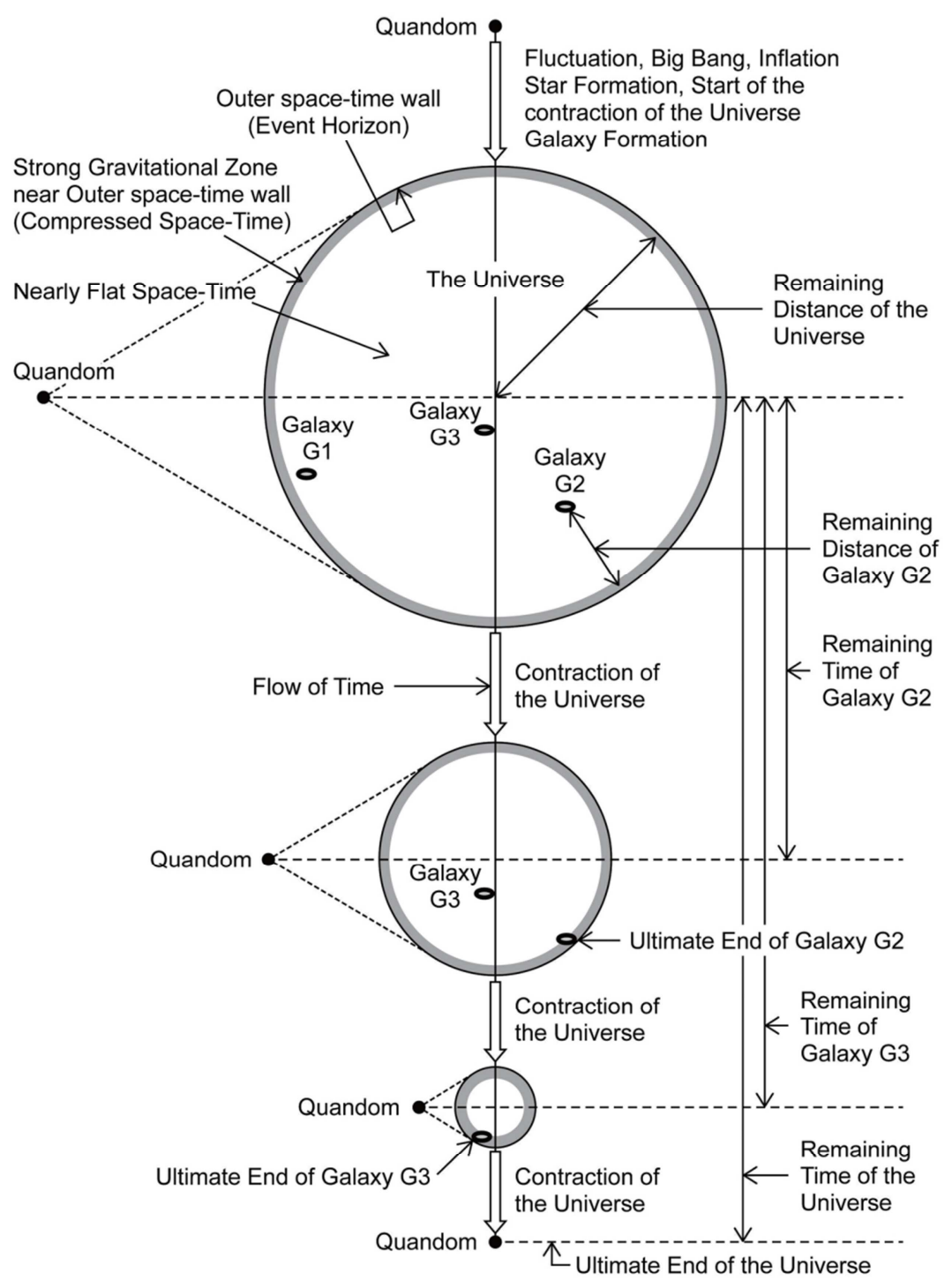

Figure 1. The Universe's structure: This figure depicts the Universe's life starting from its birth at the top to its ultimate end at the bottom, using a cross-section of the Universe. Because the quandom outside the Universe has no distance and time, it is represented by a small black circle. Space-time is more drastically compressed in the strong gravitational zone near the outer space-time wall (the grey area) than in flat space-time (the white area). 


\subsection{The Ultimate End of the Universe}

Although it looks as if the Universe is expanding, it is actually being gradually pulled back to the outer space-time wall, and its ultimate end comes eventually when the whole Universe is swallowed by the outer space-time wall. Though not a three-dimensional analogy, consider a circular rubber membrane as a two-dimensional analogy. If the circular rubber membrane's circumference is being rolled up equally, the membrane's circumference becomes thicker and thicker as it becomes shorter in length, and the area in the middle of the membrane becomes smaller and smaller as the circumference moves towards the center. Finally, the membrane becomes a rubber lump. Similarly, the Universe's circumference is being drawn into the outer space-time wall, and once the distance from the outer space-time wall to the center of the Universe falls below the limit length of matter the outer space-time wall becomes a single point and the Universe disappears (Fig. 1).

Observed from the inside of such a galaxy swallowed by the outer space-time wall, it can be confirmed up until its last moment that the Universe's isotropy is valid and the Universe's expansion speed increases. As the galaxy approaches the outer space-time wall, it will be observed that galaxies close to it fade away increasingly fast, and as the galaxy comes even closer to the outer space-time wall, stars will accelerate and fade away one by one starting from the most distant stars in the galaxy. Ultimately, the observer will reach the speed of light and on that moment be swallowed by the outer space-time wall, as a phase transition occurs marking the end of the observer's existence in the Universe. However, some entities may be swallowed by an inner space-time wall, in other words a black hole, before that, but ultimately the black hole will also be absorbed by the outer space-time wall.

\subsection{The Remaining Distance and the Remaining Time}

Each piece of matter gained a certain distance from the outer space-time wall and the corresponding time. Hereinafter, this distance and time will be called the "remaining distance" and "remaining time," respectively. The remaining distance represents the distance from matter to the closest outer space-time wall. The remaining time represents the period of time until the remaining distance of the matter becomes nil and it is absorbed by the outer space-time wall. The Universe's remaining distance and remaining time can be regarded as its radius and remaining life, respectively. The Milky Way Galaxy's remaining time is the time until the ultimate end of the galaxy, and the difference between the remaining distance of the Milky Way Galaxy and that of the Universe indicates where the Milky Way Galaxy is within the Universe. It can be argued that the farthest stars currently observed existed in the past, and must have disappeared already if the Universe is contracting. If the remaining distance of the Universe and that of the Milky Way Galaxy and the contracting speed of the Universe were known, then also the remaining time of both the Universe and the Milky Way Galaxy could be estimated.

It is considered probable that heat radiation is emitted from the outer space-time wall like heat radiation is emitted from the surface of a black hole. If this is true, the outer space-time wall may be the source of cosmic microwave background radiation. Furthermore, it is highly likely that what is regarded as dark energy may really be energy emitted from the outer space-time wall, and what is regarded as dark matter may really be gravitational force exerted by the outer space-time wall. If the Universe is a space-time bubble created in a quandom, it is not unreasonable to think that other similar bubbles or universes may exist. Likewise, if the inside of a sufficiently large black hole in the Universe is also a quandom, space-time bubbles may have been created and smaller universes may have been born there. In reverse, it is also possible that the quandom outside of our Universe itself may be a black hole created in a larger universe outside of that quandom. Therefore, it is not only conceivable that there may be or may have been brother and sister universes parallel to our Universe, but also that universes and quandoms may exist hierarchically in multiple layers like a matryoshka doll.

\section{Discussion}

The crucial point of this paper, which can be deduced from the theory, is that the Universe is not expanding, but is contracting. From this viewpoint, it is to be mentioned that $\mathrm{C}$. Tsagas of Greece pointed out that the "apparent accelerated expansion" of the Universe is just an illusion caused by our relative motion to the rest of the Universe [10]. Although the Tsagas's rationale that denies the accelerated expansion of the Universe is somewhat different from what is presented in our paper, it would be a time to address questions regarding the relative and multi-dimensional movements of the components of the Universe. It is also to be noted that Shafieloo and his coworkers investigated the so-called cosmic expansion using the Constitution SN Ia sample, baryon acoustic oscillation and cosmic microwave background data. They concluded that cosmic acceleration may have already peaked and that we are currently witnessing its slowing down [11]. They further pointed out that the effect they observed could correspond to dark energy decaying into dark matter [11].

\section{Conclusion}

A novel concept of "quandom" is proposed in this report, in which the current status and the fate of the Universe are explained. According to this concept, the Universe is not expanding but contracting from its boundary, while satisfactorily explaining the observed results of the Universe's isotropy and apparent expansion. This concept will help understand the present status and the ultimate fate of the Universe. Experimental researches are needed to prove this concept. 


\section{References}

[1] E. Hubble, "A relation between distance and radial velocity among extra-galactic nebulae," Proc. Natl. Acad. Sci. USA, vol. 15 , pp. 168-173, 1929.

[2] K. Kamada, "Inflationary cosmology and the standard model Higgs with a small Hubble-induced mass," Phys. Lett. B, vol. 742, pp. 126-135, 2015.

[3] B. P. Schmidt, R. P. Kirshner, and R. G. Eastman, "Expanding photospheres of type II supernovae and the extragalactic distance scale,” Astrophys. J., vol. 395, pp. 366-386, 1992.

[4] A. Addazi, S. Capozziello, S. Odintsov, "Born-lnfeld condensate as a possible origin of neutrino masses and dark energy,” Phys. Lett. B, vol. 760, pp. 611-616, 2016.

[5] D. Comelli, M. Pietroni, and A. Riotto, "Dark energy and dark matter,” Phys. Lett. B, vol. 571, pp. 115-120, 2003.

[6] K. Nozari, N. Behrouz, "An interacting dark energy model with nonminimal derivative coupling," Phys. Dark Universe, vol. 13, pp. 92-110, 2016.

[7] K. Bamba, S. Capozziello, S. Nojiri, and S. D. Odintsov, "Dark energy cosmology: the equivalent description via different theoretical models and cosmography tests," Astrophys. Space Sci., vol. 342, pp. 155-228, 2012.

[8] P.-H. Chavanis, "The logotropic dark fluid as a unification of dark matter and dark energy," Phys. Lett. B, vol. 758, pp. 59-66, 2016.

[9] C. M. Ho, and S. D. H. Hsu, "Astrophysical constraints on dark energy,” Astroparticle Phys, vol. 74, pp. 47-50, 2016.

[10] C. G. Tsagas, "Peculiar motions, accelerated expansion, and the cosmological axis," Phys. Rev. D, vol. 84, pp. 063503, 2011.

[11] A. Shafieloo, V. Sahni, and A. A. Starobinsky, "Is cosmic acceleration slowing down?" Phys. Rev. D, vol. 80, pp. 101301 (R), 2009. 